\title{
A organização da escolaridade em ciclos no contexto do Ensino Fundamental de Nove Anos: reflexões e perspectivas ${ }^{1}$
}

\author{
Organization of the schooling in cycles of nine year long \\ Primary Education: thoughts and perspectives
}

\section{Jefferson Mainardes ${ }^{2}$ Silvana Stremel ${ }^{3}$}

\section{Resumo:}

Este artigo discute aspectos relacionados à organização da escolaridade em ciclos no contexto da implantação do Ensino Fundamental de Nove Anos. Apresenta uma contextualização da proposição dos ciclos nos três primeiros anos do Ensino Fundamental, as possibilidades e desafios de sua implantação e ainda o papel das secretarias de educação, escolas e profissionais da educação. Argumenta-se que a discussão sobre ciclos no contexto atual precisa ir além das discussões sobre a eliminação da reprovação e da aprendizagem enquanto um processo contínuo, pois a implantação dos ciclos nos anos iniciais pressupõe uma revisão ampla e profunda da concepção de currículo, avaliação, metodologia, organização do sistema de ensino, gestão da escola, relação escola-comunidade, formação continuada de professores, entre outros aspectos.

Palavras-chave: Ensino Fundamental; Ciclos; Política Educacional.

\begin{abstract}
:
This article discusses issues related to the organization of schooling in cycles in the context of the implementation of nine-year long Basic Education in Brazil. It presents an overview of the proposition of cycles (without retention) in the first three years of Primary school, the possibilities and challenges of its implementation and also the role of the education departments, schools and practitioners. It argues that the discussion of cycles in the current context needs to go beyond the discussions on the elimination of retention and that learning is a continuous process since the implementation of cycles in the early years requires a broad and deep review of curriculum, assessment, methodology, organisation of the education system, school management, schoolcommunity relationship and continuing teacher training, among other aspects.
\end{abstract}

Keywords: Primary Education; Cycles; Education Policy.

1 Agências financiadoras: CNPq e Capes.

2 Professor Doutor do Programa de Pós-Graduação em Educação da Universidade Estadual de Ponta Grossa. E-mail: jefferson.m@uol.com.br 3 Mestre em Educação pela Universidade Estadual de Ponta Grossa. E-mail: silvanastremel@gmail.com 


\section{Introdução}

A implantação do Ensino Fundamental de Nove Anos representa um avanço significativo na oferta educacional, principalmente para as classes trabalhadoras que, historicamente, têm tido um acesso mais limitado ao processo de escolarização, seja em termos do acesso, da permanência e do resultado (igualdade de conhecimentos adquiridos na escola e sucesso escolar). Entendemos que se trata de uma conquista histórica forjada tanto no contexto do desenvolvimento econômico e sociocultural quanto na luta social pela ampliação do acesso e da expansão da escolaridade obrigatória.

A implantação dessa medida requer a alteração de diversos aspectos relacionados à reorganização do tempo, do currículo, da avaliação, da organização da escola, das práticas pedagógicas. Nos limites deste artigo, destacaremos que a forma como o tempo de escolarização é distribuído e organizado ao longo dos nove anos do Ensino Fundamental constitui-se em uma questão essencial. No sistema educacional brasileiro, as redes de ensino possuem autonomia para decidir sobre a organização do ensino em séries anuais, períodos semestrais, ciclos, alternância regular de períodos de estudos, grupos não seriados (Art. 23 da Lei n 9394/96) (BRASIL, 1996). Além disso, o $\S 2^{\circ}$ do Art. 32 da mesma Lei indica que os estabelecimentos que utilizam a progressão regular por série podem adotar, no Ensino Fundamental, o regime de progressão continuada. Com isso, a reprovação é eliminada em alguns anos ou séries.

No Brasil, nos últimos 25 anos, diversas redes de ensino implementaram diferentes modalidades de políticas de ciclos (Ciclo Básico, Ciclos de Aprendizagem, Ciclos de Formação, Regime de Progressão Continuada, Ciclo Inicial de Alfabetização, Bloco Inicial de Alfabetização, etc. $)^{4}$ com o intuito de diminuir os índices de reprovação e evasão. Em diversas redes de ensino, a implantação dos ciclos foi marcada pela proposição de alterações mais profundas no sistema educacional, com a reorganização do tempo e do sistema de promoção dos alunos, do currículo, da avaliação, das metodologias de ensino, da gestão da escola e da ampliação da participação da comunidade na vida da escola. Por outro lado, em outras redes de ensino as mudanças foram menos substanciais.

Nesse artigo, buscamos situar o debate atual sobre a eliminação da reprovação nos três primeiros anos de escolaridade, indicar algumas mudanças que a ampliação do Ensino Fundamental de Nove Anos tem causado na política de ciclos e ainda discutir as principais implicações dessa medida.

\section{Contextualização da proposta de implantação dos ciclos no Ensino Fundamental de Nove Anos}

A discussão sobre a eliminação da reprovação nos anos iniciais de escolarização não é nova. As pesquisas históricas sobre as políticas de não reprovação no Brasil indicam que desde a década de 1910 já se discutia a viabilidade de eliminar a reprovação no ensino primário, por meio da "promoção em massa", em virtude dos elevados índices de reprovação e falta de vagas (ALMEIDA JÚNIOR, 1957, BARRETTO; MITRULIS, 1999, 2001; MAINARDES, 1998, 2001, 2007, 2009a). Na década de 1950, a discussão sobre a promoção automática foi bastante intensa (TEIXEIRA, 1954; KUBITSCHEK, 1957; ALMEIDA JÚNIOR, 1957; LEITE, 1959), sendo que as experiências pioneiras de eliminação da reprovação ocorreram a partir da década de 1950 (ARELARO, 1988; BARRETTO, 1999, 2001; MAINARDES, 2007, 2009a). A partir da década de 1980, diversas redes implantaram o Ciclo Básico de Alfabetização, no qual a reprovação do $1^{\circ}$ para o $2^{\circ}$ ano de escolaridade era eliminada. A partir dos anos 1990, diversas modalidades de ciclos mais longos e com propostas mais complexas foram

4 Os pesquisadores que estudam essa temática destacam que há diferenças entre as modalidades de ciclos existentes. Algumas modalidades propõem ciclos mais longos, a eliminação da reprovação em todos os anos e mudanças mais radicais no sistema educacional. Já outras propõem ciclos mais curtos, a reprovação ao final dos ciclos e mudanças menos substanciais no sistema de ensino. Assim, há diferenças entre o Regime de Progressão Continuada e as diferentes propostas de ciclos existentes. Freitas (2003), Mainardes (2007), Jacomini (2010) discutem as diferenças entre as propostas de ciclos e o Regime de Progressão Continuada. 
implementadas (Ciclos de Aprendizagem, Ciclos de Formação, Regime de Progressão Continuada, Ciclo Inicial de Alfabetização, Bloco Inicial de Alfabetização, etc.).

Segundo dados do MEC/Inep, no ano de 2006, 9,72\% das escolas do Ensino Fundamental estavam organizadas em ciclos (18,17\% das matrículas) e 7,16\% possuíam séries e ciclos (14,5\% das matrículas). A implantação do Ensino Fundamental de Nove Anos reacendeu a discussão da organização da escolaridade em ciclos, principalmente nos três primeiros anos de escolaridade. Deve-se destacar que diversas propostas de organização da escolaridade em ciclos, implementadas a partir da segunda metade dos anos 1990, já contemplavam a inclusão de crianças de seis anos no primeiro ciclo do Ensino Fundamental.

Os pareceres CNE/CEB n 7/2007 e 4/2008 indicam e justificam a relevância de se garantir que os três primeiros anos sejam entendidos como um processo contínuo. (BRASIL, 2007, 2008). As Diretrizes Curriculares do Ensino Fundamental (Resolução $n^{\circ} 7$, de 14 de dezembro de 2010) também mencionam os ciclos enquanto uma possibilidade de organização do Ensino Fundamental (BRASIL, 2010). A retomada e o revigoramento dessa alternativa têm sido influenciados por uma série de fatores:

a) Diversas redes de ensino brasileiras já vivenciam (ou já vivenciaram) a organização do Ensino Fundamental em ciclos, principalmente nos anos iniciais da escolarização. Conforme já indicado, 18,17\% dos alunos matriculados no Ensino Fundamental em 2006 estavam dentro do sistema de ciclos. Desse modo, as redes de ensino brasileiras já possuem um nível de experiência acumulada sobre a implantação de diferentes modalidades de ciclos. Há, também, um significativo número de pesquisas e publicações que apresentam resultados sobre a implementação dessas políticas ${ }^{5}$. De modo geral, os estudos indicam que a implantação dos ciclos é complexa, uma vez que traz implicações para o currículo, a avaliação, as metodologias, a organização e gestão da escola. Além disso, pressupõe mudanças na infraestrutura das escolas, formação continuada de professores, além de uma série de medidas complementares, sem as quais a proposta dos ciclos fica prejudicada. Trata-se de uma mudança na estrutura do sistema de escolarização que traz diversas implicações para o trabalho docente, organização da escola, relação escola-comunidade e que demanda investimentos de natureza diversa, algo que é atendido de forma variada e algumas vezes descontínua nos diferentes contextos nos quais os ciclos são implantados.

b) Os dados do MEC/Inep indicam que uma média de 3,0 a 3,5\% dos alunos do ano inicial (6 anos de idade) são reprovados já no primeiro ano de escolaridade (Tabela 1 e Tabela 2). Os dados do ano de 2009 (Brasil) indicam que no ano inicial estavam matriculadas 2.528.631 alunos. A taxa de reprovação na rede federal foi de $3,8 \%$; na rede estadual 1,6\%, na rede municipal 3,4\% e na rede particular 2,1\%. A média nacional foi de 3,0\%, ou seja, mais de 74.000 crianças foram reprovadas já no primeiro ano de escolaridade, aos seis anos de idade. Os índices mais elevados foram os seguintes: Rio de Janeiro (9,3\%); Piauí (8,7\%); Paraíba (6,6\%); Bahia (5,2\%); Pará (4,7\%); Amazonas (4,4\%) e Ceará (4,3\%). Os índices mais baixos foram os seguintes: Amapá (0,4\%); Mato Grosso do Sul (0,5\%); Rondônia (0,8\%); Tocantins (0,8\%); Santa Catarina (0,8\%); Rio Grande do Sul (0,9\%) e Mato Grosso (0,9\%). No ano de 2010, 2,6\% dos alunos do ano inicial foram reprovados e 1,6\% abandonaram. A análise dos dados de 2010 evidenciou que as taxas de reprovação e evasão das escolas rurais são mais elevadas, sendo 4,4\% (reprovação - total rural) e 3,1\% (abandono - total rural). Já os dados das escolas urbanas são de 2,1\% (reprovação) e 1,4\% (abandono). Esses dados indicam que as escolas situadas na área rural demandam maior atenção por parte dos gestores educacionais. Deve-se destacar, também, que as taxas de reprovação e evasão no ano inicial estão diminuindo: de 3,0\% em 2009 para 2,6\% em 2010 (reprovação) e de 2,1\% em 2009 para 1,6\% em 2010 (evasão).

5 Há diversos trabalhos que apresentam sínteses sobre os ciclos no Brasil (MAINARDES, 2009a), bem como sobre as pesquisas que têm sido realizadas no Brasil sobre essa temática (SOUSA et al., 2003; BARRETTO; SOUSA, 2004; GOMES, 2004; SOUSA; BARRETTO, 2004; MAINARDES, 2006, 2008, 2009b). Sugerimos também o site <http://www.uepg.br/gppepe> que apresenta uma lista de mais de 200 teses e dissertações sobre o tema, defendidas no período de 2000 a 2011. 
Tabela 1 - Número de alunos aprovados, reprovados e que deixaram de frequentar a escola e taxa de aprovação, reprovação e abandono, no Ano Inicial do Ensino Fundamental, por ano - Brasil

\begin{tabular}{|c|c|c|c|c|c|c|c|}
\hline \multirow[b]{2}{*}{ Ano } & \multirow{2}{*}{$\begin{array}{l}\text { Total de alunos } \\
\text { matriculados }\end{array}$} & \multicolumn{3}{|c|}{$\begin{array}{c}\text { Número de alunos aprovados, reprovados e que } \\
\text { deixaram de frequentar a escola no Ano Inicial do } \\
\text { Ensino Fundamental }\end{array}$} & \multicolumn{3}{|c|}{$\begin{array}{l}\text { Taxa de aprovação, reprovação e abandono no Ano } \\
\text { Inicial do Ensino Fundamental }\end{array}$} \\
\hline & & Aprovados & Reprovados & $\begin{array}{l}\text { Alunos que } \\
\text { deixaram de } \\
\text { frequentar a } \\
\text { escola }\end{array}$ & Aprovação & Reprovação & Abandono \\
\hline 2004 & 739.714 & 673.274 & 29.989 & 40.907 & 90,5 & 4,0 & 5,5 \\
\hline 2005 & 908.052 & 813.360 & 42.964 & 50.034 & 89,5 & 4,6 & 5,9 \\
\hline 2006 & 1.336 .992 & - & - & - & - & - & - \\
\hline 2007 & 1.957 .337 & 1.728 .339 & 74.096 & 55.334 & 93,0 & 4,0 & 3,0 \\
\hline 2008 & 2.266 .667 & 2.015 .202 & 74.471 & 54.743 & 94,0 & 3,5 & 2,5 \\
\hline 2009 & 2.528 .631 & - & - & - & 94,9 & 3,0 & 2,1 \\
\hline
\end{tabular}

Fonte: MEC/Inep.

Tabela 2 - Taxa de aprovação, reprovação e abandono, no Ano Inicial do Ensino Fundamental, Zonas Rural e Urbana, por ano e dependểncia administrativa - Brasil

\begin{tabular}{|c|c|c|c|c|c|c|c|c|c|c|}
\hline \multirow{3}{*}{ Ano } & \multirow{3}{*}{$\begin{array}{l}\text { Dependência } \\
\text { Administrativa }\end{array}$} & \multicolumn{9}{|c|}{ Taxas } \\
\hline & & \multicolumn{3}{|c|}{ Aprovação } & \multicolumn{3}{|c|}{ Reprovação } & \multicolumn{3}{|c|}{ Abandono } \\
\hline & & $\begin{array}{l}\text { Zona } \\
\text { Rural }\end{array}$ & $\begin{array}{c}\text { Zona } \\
\text { Urbana }\end{array}$ & Total & $\begin{array}{l}\text { Zona } \\
\text { Rural }\end{array}$ & $\begin{array}{c}\text { Zona } \\
\text { Urbana }\end{array}$ & Total & $\begin{array}{l}\text { Zona } \\
\text { Rural }\end{array}$ & $\begin{array}{c}\text { Zona } \\
\text { Urbana }\end{array}$ & Total \\
\hline \multirow{5}{*}{2007} & Federal & - & 94,8 & 94,8 & - & 4,8 & 4,8 & - & 0,4 & 0,4 \\
\hline & Estadual & 92,4 & 94,2 & 94,0 & 4,0 & 2,5 & 2,6 & 3,6 & 3,3 & 3,4 \\
\hline & Municipal & 87,3 & 93,5 & 92,1 & 8,4 & 3,5 & 4,6 & 4,3 & 3,0 & 3,3 \\
\hline & Particular & 94,1 & 97,4 & 97,4 & 4,6 & 2,0 & 2,0 & 1,3 & 0,6 & 0,6 \\
\hline & Total & 87,7 & 94,2 & 93,0 & 8,0 & 3,1 & 4,0 & 4,3 & 2,7 & 3,0 \\
\hline \multirow{5}{*}{2008} & Federal & - & 96,8 & 96,8 & - & 3,0 & 3,0 & - & 0,2 & 0,2 \\
\hline & Estadual & 92,0 & 95,9 & 95,6 & 4,5 & 1,6 & 1,9 & 3,5 & 2,5 & 2,5 \\
\hline & Municipal & 88,9 & 94,1 & 92,9 & 7,0 & 3,3 & 4,1 & 4,1 & 2,6 & 3,0 \\
\hline & Particular & 94,3 & 97,7 & 97,7 & 4,6 & 1,8 & 1,8 & 1,1 & 0,5 & 0,5 \\
\hline & Total & 89,2 & 95,0 & 94,0 & 6,8 & 2,8 & 3,5 & 4,0 & 2,2 & 2,5 \\
\hline \multirow{5}{*}{2009} & Federal & - & 96,9 & 95,9 & - & 3,8 & 3,8 & - & 0,3 & 0,3 \\
\hline & Estadual & 93,2 & 96,5 & 96,2 & 3,4 & 1,6 & 1,6 & 3,4 & 2,0 & 2,2 \\
\hline & Municipal & 90,9 & 95,1 & 94,2 & 5,7 & 2,8 & 3,4 & 3,4 & 2,1 & 2,4 \\
\hline & Particular & 89,2 & 97,5 & 97,4 & 9,5 & 2,0 & 2,1 & 1,3 & 0,5 & 0,5 \\
\hline & Total & 91,1 & 95,7 & 94,9 & 5,6 & 2,5 & 3,0 & 3,3 & 1,8 & 2,1 \\
\hline \multirow{5}{*}{2010} & Federal & 92,3 & 96,2 & 96,2 & 0 & 3,6 & 3,6 & 7,7 & 0,2 & 0,2 \\
\hline & Estadual & 94,2 & 97,7 & 97,5 & 2,8 & 0,9 & 1,1 & 3,0 & 1,4 & 1,4 \\
\hline & Municipal & 92,4 & 95,9 & 95,1 & 4,5 & 2,5 & 2,9 & 3,1 & 1,6 & 2,0 \\
\hline & Particular & 90,8 & 97,5 & 97,5 & 8,2 & 2,0 & 2,1 & 1,0 & 0,5 & 0,4 \\
\hline & Total & 92,5 & 96,5 & 95,8 & 4,4 & 2,1 & 2,6 & 3,1 & 1,4 & 1,6 \\
\hline
\end{tabular}

Fonte: MEC/Inep.

A constatação desses índices de reprovação se dá em um contexto histórico no qual as pesquisas internacionais e nacionais já demonstraram que a reprovação, principalmente nos anos iniciais, não é benéfica e que os alunos que reprovam em um ano não apresentam, nos anos posteriores, melhor desempenho que os alunos aprovados (HOLMES; MATTHEWS, 1984; SMITH; SHEPARD, 1987; HOLMES, 1989; CRAHAY, 1996). Os 
resultados dessas pesquisas, aliados a outros aspectos, têm fundamentado a ideia de que a política de ciclos é viável e adequada para a escola pública. Entre os aspectos que têm contribuído para tal fundamentação destacamos os seguintes: a) a compreensão de que a aprendizagem precisa ser entendida como um processo contínuo, o que dispensaria interrupções desnecessárias, a exemplo do que faz a reprovação anual; e b) a construção de um sistema educacional inclusivo e democrático pressupõe a implementação de políticas que visem à superação de práticas que, historicamente, têm levado à exclusão de alunos do processo de escolarização. A concepção da aprendizagem como um processo contínuo tem sido particularmente destacada no processo de alfabetização, uma vez que esse processo dificilmente se dá apenas em um ano escolar. Assim, garantir um tempo maior para que as crianças da classe trabalhadora possam obter a apropriação da leitura e da escrita é uma questão essencial e que deve ser garantida a todas as crianças de modo efetivo.

Conforme já mencionado, com a implantação do Ensino Fundamental de Nove Anos, o CNE passou a recomendar que os anos iniciais fossem transformados em um ciclo único (ciclo da infância), sem reprovação. Essa proposta foi também incorporada pelo Ministério da Educação. Essas recomendações reacenderam o debate sobre os ciclos e a eliminação da reprovação. No entanto, observa-se que a discussão atual, no contexto do Ensino Fundamental de Nove Anos, tem evidenciado mais a questão da não reprovação e da alfabetização como processo contínuo. Assim, as implicações dessa medida para o currículo, avaliação, metodologias de ensino, organização e gestão da escola, formação continuada de professores tem sido menos evidenciadas. Nesse contexto, argumentamos que as discussões sobre a implantação dos ciclos nos três anos iniciais precisariam incorporar as implicações pedagógicas e políticas que têm sido pautadas nas discussões sobre a política de ciclos, desde a década de 1980, quando o Ciclo Básico de Alfabetização passou a ser implementado em diferentes redes de ensino. Assim, é necessário ir muito além das discussões sobre a eliminação da reprovação e da aprendizagem enquanto um processo contínuo, pois a implantação dos ciclos nos anos iniciais pressupõe uma revisão de toda a concepção de currículo, avaliação, metodologia, organização do sistema de ensino, gestão da escola, relação escola-comunidade, formação continuada de professores, entre outros aspectos.

De modo geral, tanto o campo oficial quanto o pedagógico ${ }^{6}$ admitem que a política de ciclos é uma proposta viável e positiva, mas não deixam de indicar que diversos cuidados precisam ser tomados para evitar que essa proposta seja mal compreendida ou implantada de forma inadequada, o que poderia levar a prejuízos para os alunos. Entre esses cuidados podemos citar a formação continuada de professores, o acompanhamento às escolas e professores, a elaboração de projetos consistentes pelas secretarias, a implantação de medidas complementares (por exemplo, tempo para complementação de estudos, especialmente para alunos que necessitam de maior tempo para a apropriação dos conteúdos), a ampliação de recursos financeiros que viabilize o aumento dos professores, a aquisição de materiais pedagógicos diversificados, etc.

\section{Ciclos no Ensino Fundamental de Nove Anos: desafios e possibilidades}

A implantação dos ciclos nos Ensino Fundamental, principalmente nos três primeiros anos do processo de escolarização cria demandas para o Conselho Nacional de Educação, Ministério de Educação e secretarias, escolas, profissionais da educação e pesquisadores. Até o presente, o CNE e o MEC têm assumido a validade da eliminação da reprovação e destacado a autonomia das redes de ensino e das escolas para organizar a distribuição do tempo do Ensino Fundamental em séries ou ciclos. Assim, caberá às redes de ensino que

6 Para Bernstein (1996), o campo oficial é criado e dominado pelo Estado e suas agências, autoridades ou departamentos, através de agentes especializados em produzir o discurso pedagógico oficial. 0 campo pedagógico é constituído por pedagogos em escolas, faculdades e setores de educação de universidades com suas pesquisas, periódicos e jornais especializados, fundações privadas de pesquisa. Segundo Bernstein (1996) esses dois campos têm um efeito sobre o discurso pedagógico independentemente do um do outro, portanto, disputarão espaços e influências na configuração do discurso pedagógico. 
ainda adotam o sistema seriado, decidir sobre o modelo de ciclos que pretendem adotar. $\mathrm{O}$ envolvimento e a participação dos profissionais da educação que atuam nas escolas e de pesquisadores podem ser indicados como estratégias altamente recomendáveis.

O Parecer CNE/CEB n 7/2007 sugere que o Ensino Fundamental seja organizado em "ciclos pedagógicos". O Parecer CNE/CEB n 4/2008 (BRASIL, 2008) destaca os seguintes aspectos:

a) que o Ensino Fundamental é um "novo Ensino Fundamental, que exige um projeto político-pedagógico próprio para ser desenvolvido em cada escola" (BRASIL, 2008, p. 2);

b) a organização do Ensino Fundamental com nove anos de duração supõe a reorganização da Educação Infantil, particularmente da Pré-Escola, destinada agora, a crianças de 4 e 5 anos de idade;

c) o terceiro período da Pré-Escola não pode se confundir com o primeiro ano do Ensino Fundamental, pois esse primeiro ano é agora parte integrante de um ciclo de três anos de duração, que poderia ser denominado de "ciclo da infância";

d) mesmo que o sistema de ensino ou a escola faça a opção pelo sistema seriado, há necessidade de se considerar esses três anos iniciais como um bloco pedagógico ou ciclo sequencial de ensino;

e) entende-se que a alfabetização dar-se-á nos três primeiros anos iniciais do Ensino Fundamental.

As justificativas e argumentações citadas acima são muito claras e consistentes. Apesar disso, as discussões sobre a implantação dos ciclos, no contexto da implantação do Ensino Fundamental de Nove Anos diferenciam-se das discussões que integravam a implantação dos ciclos no período anterior à implantação do Ensino Fundamental de Nove Anos. No período anterior, diversas redes de ensino, desde os anos 1990, já estavam incluindo crianças de seis anos de idade no Ensino Fundamental. Naquele contexto,

a implantação dos ciclos almejava criar um sistema de ciclos que abrangesse os nove anos do Ensino Fundamental ou, pelo menos, os cinco primeiros anos. Além disso, de modo geral, a implantação dos ciclos envolvia não apenas a alteração do sistema de promoção, mas também mudanças no currículo, na avaliação, nas metodologias de ensino. As redes optavam por modalidades de ciclos, tais como: Ciclos de Aprendizagem, Ciclos de Formação, Ciclo Inicial, Bloco Inicial, Progressão Continuada, entre outros. Já no contexto da implantação do Ensino Fundamental de Nove Anos, tem-se enfatizado mais a implantação dos ciclos apenas nos três primeiros anos. Assim, há o risco de que as propostas de implantação de ciclos passem a priorizar mais a não reprovação dos alunos de seis e sete anos de idade (o que é um aspecto positivo) e deixem de enfatizar os ciclos como uma possibilidade de uma alteração mais profunda da organização da escola. Do nosso ponto de vista, não basta apenas eliminar a reprovação. É essencial garantir a todos os alunos a aprendizagem da forma mais ampla, exitosa e no menor tempo possível (KLEIN, 2003). Para que isso ocorra, uma série de decisões precisa ser tomada pelas secretarias de educação e escolas no sentido de definir a sistemática de promoção dos alunos (duração dos ciclos, que podem abranger os demais anos do Ensino Fundamental e não apenas os três primeiros), uma proposta curricular, a sistemática de avaliação, as estratégias de formação continuada, etc.

\section{A implantação de ciclos e o papel das secretarias de educação, das escolas e dos profissionais da educação}

Conforme já mencionado, as redes de ensino possuem autonomia para decidir sobre a organização do sistema de ensino. No contexto da proposta da implantação dos ciclos no Ensino Fundamental de Nove Anos, seria recomendável que as secretarias de educação analisassem as diferentes possibilidades de reorganização do tempo do Ensino Fundamental; as diferentes modalidades de ciclos; as implicações dos ciclos para o currículo, avaliação, metodologias, gestão da escola, formação de professores; as necessidades de infraestrutura, etc. Conforme já mencionado, há um número significativo de documentos 
oficiais de secretarias de educação, bem como de pesquisas sobre os ciclos que podem subsidiar as discussões no âmbito das secretarias. No entanto, deve-se destacar que o envolvimento dos profissionais que atuam nas escolas (professores, pedagogos, diretores) é um aspecto crucial para o delineamento de políticas de ciclos, uma vez que, de modo geral, a cultura da seriação e da reprovação são bastante arraigadas nas crenças e concepções dos profissionais que atuam na escola. Em termos ideais, a política de ciclos precisaria ser construída de acordo com as características da rede, dos professores e da infraestrutura disponível. A criação de espaços de discussão e de formação de professores para lidar com as mudanças e desafios dos ciclos é uma das ações que poderia ser privilegiada. Estratégias mais amplas de acompanhamento também são necessárias, principalmente em redes de ensino que nunca vivenciaram experiências de não reprovação. As estratégias mais amplas de acompanhamento referem-se à importância de criar espaços de discussão e formação dos profissionais da educação e ainda a avaliação do desempenho dos alunos nos ciclos.

A atuação da equipe pedagógica das escolas assume uma especial relevância para o sucesso dos ciclos. De modo geral, cabe à equipe pedagógica das escolas fazer a mediação entre a proposta de ciclos da rede e a sua efetivação na escola e nas salas de aula. Nesse aspecto, é importante destacar que as políticas educacionais e curriculares não são meramente implementadas pelos profissionais da educação que atuam no contexto da prática. Tais políticas estão sujeitas a processos de recriação e reinterpretação, bem como à criação de ajustamentos secundários (BALL, 1994). Isso significa que os professores interagem com as políticas e propostas oficiais e desenvolvem alternativas para implementá-las. Assim, dependendo do contexto global da escola, das condições objetivas de infraestrutura e condições de trabalho, das ações coletivas realizadas pela equipe pedagógica e professores, o processo de implementação dessas políticas será mais ou menos facilitado. O contexto dos resultados está relacionado ao contexto da prática que, por sua vez, é condicionado por fatores contextuais objetivos: o macrossistema econômico, político, social e cultural; a escola e suas condições objetivas; a equipe que atua na escola; o nível de conhecimento e de apropriação da política proposta pelos profissionais que atuam na escola, entre outros fatores.

A implantação dos ciclos, ainda que apenas nos três primeiros anos do Ensino Fundamental traz diversos desafios para os professores. O primeiro desafio é a necessidade de conhecer o projeto de ciclos proposto para a rede, o significado dessa política e as implicações dessa política para o seu trabalho. Em muitos casos, os professores têm acesso apenas a informações básicas, voltadas à prática, sendo necessária a busca individual ou a auto-organização dos docentes, com o apoio da equipe da escola, de grupos de escolas ou de sindicatos para a ampliação do conhecimento e das oportunidades de estudo e discussão sobre os ciclos. Um segundo desafio diz respeito às implicações pedagógicas dos ciclos, uma vez que a implantação de ciclos pressupõe uma alteração significativa nos processos de avaliação e desenvolvimento curricular que eram empregados pelos professores no regime seriado. Os professores precisarão, por exemplo, trabalhar com os princípios da avaliação formativa e da pedagogia diferenciada para atender a heterogeneidade das classes. Para isso, eles necessitarão de apoio da equipe da escola e das secretarias de educação, que podem criar espaços de formação sobre essas questões. As dificuldades e dúvidas serão muitas, principalmente em uma fase inicial e o negligenciamento dessas questões poderá ter um impacto negativo nos processos de aprendizagem dos alunos, bem como do interesse dos professores pelos ciclos.

Finalmente, acreditamos que os pesquisadores podem contribuir não apenas para a construção do conhecimento sobre ciclos e para a elaboração de sínteses de resultados obtidos por meio de sua implementação, mas, sobretudo, para a qualificação das redes de ensino, dos professores e dos futuros professores para enfrentar os desafios dos ciclos e a implantação de políticas de ciclos que sejam mais participativas e resultado de reflexões coletivas sobre os processos de ensino-aprendizagem e formação dos alunos. 


\section{Referências Bibliográficas}

ALMEIDA JÚNIOR, Antônio. Repetência ou promoção automática? Revista Brasileira de Estudos Pedagógicos, Rio de Janeiro, v. 27, n. 65, p. 3-15, jan./mar. 1957.

ARELARO, Lisete Regina Gomes. A (ex)tensão do ensino básico no Brasil: o avesso de um direito democrático. 1988. 322 f. Tese (Doutorado em Educação) - Faculdade de Educação, Universidade de São Paulo, São Paulo, 1988.

BALL, Stephen J. Education reform: a critical and post structural approach. Buckingham: Open University Press, 1994.

BARRETTO, Elba Siqueira de Sá; MITRULIS, Eleny. Os ciclos escolares: elementos de uma trajetória. Cadernos de Pesquisa, São Paulo, n. 108, p. 27-48, nov. 1999.

BARRETTO, Elba Siqueira de Sá; MITRULIS, Eleny. Trajetória e desafios dos ciclos escolares no país. Estudos avançados/USP, São Paulo, v. 15, n. 42, p. 103-140, maio/ago. 2001.

BARRETTO, Elba Siqueira de Sá; SOUSA, Sandra Zákia. Estudos sobre ciclos e progressão escolar no Brasil: uma revisão. Educação e Pesquisa, São Paulo, v. 30, n. 1, p. 31-50, jan./abr. 2004.

BERNSTEIN, Basil. A estruturação do discurso pedagógico: classe, códigos e controle. Petrópolis: Vozes, 1996.

BRASIL. Lei $\mathbf{n}^{\circ}$ 9394, de 20 de dezembro de 1996. Estabelece as diretrizes e bases da educação nacional. Brasília: Congresso Nacional, 1996.

BRASIL. Ministério da Educação. Conselho Nacional de Educação. Parecer CNE/CEB n 5/2007, de $\mathbf{1}^{\circ}$ de fevereiro de 2007. Consulta com base nas Leis $n^{\circ}$ 11.114/2005 e $n^{\circ} 11.274 / 2006$, que tratam do ensino fundamental de nove anos e da matrícula obrigatória de crianças de seis anos no ensino fundamental. Brasília: CNE/CEB, 2007a.

BRASIL. Ministério da Educação. Conselho Nacional de Educação. Parecer CNE/CEB n 7/2007, de 14 de abril de 2007. Reexame do Parecer CNE/CEB n 5/2007, que trata da consulta com base nas Leis n 11.114/2005 e $n^{\circ} 11.274 / 2006$, que se referem ao ensino fundamental de nove anos e à matrícula obrigatória de crianças de seis anos no ensino fundamental. Brasília: CNE/CEB, 2007b.

BRASIL. Ministério da Educação. Conselho Nacional de Educação. Parecer CNE/CEB n 4/2008, de 20 de fevereiro de 2008. Orientação sobre os três anos iniciais do ensino fundamental de nove anos. Brasília: CNE/ CEB, 2008.

BRASIL. Ministério da Educação. Conselho Nacional de Educação. Resolução CNE/CEB 7/2010. Fixa Diretrizes Curriculares Nacionais para o Ensino Fundamental de 9 (nove) anos. Diário Oficial da União, Brasília, 15 dez. 2010, Seção 1, p. 34.

CRAHAY, Marcel. Podemos lutar contra o insucesso escolar? Lisboa: Instituto Piaget, 1996. (Horizontes Pedagógicos, 59).

FREITAS, L. C. de. Ciclos, seriação e avaliação: confronto de lógicas. São Paulo: Moderna, 2003.

GOMES, Cândido Alberto. Quinze anos de ciclos no ensino fundamental: uma análise dos percursos. Revista Brasileira de Educação, Rio de Janeiro, n. 25, p. 39-52, jan./abr. 2004.

HOLMES, C. Thomas. Grade level retention effects: a meta-analysis of research studies. In: SHEPARD, Lorrie A.; SMITH, Mary Lee. Flunking grades: research and policies on retention. London: Falmer Press, 1989. p. 1633.

HOLMES, C. Thomas; MATTHEWS, Kenneth M. The effects of nonpromotion on Elementary and Junior High School Pupil: a meta-analysis. Review of Educational Research, n. 54, p. 225-236, 1984.

JACOMINI, M. A. Educar sem reprovar. São Paulo: Cortez, 2010.

KLEIN, Lígia Regina. Fundamentos teóricos - os ciclos de aprendizagem e a qualidade da escola pública. In: 
Seminário de Educação e Políticas Educacionais: qualidade da escola pública e os Ciclos de Aprendizagem. Curitiba, 2003.

KUBITSCHEK, Juscelino. Reforma do ensino primário com base no sistema de promoção automática. Revista Brasileira de Estudos Pedagógicos, Rio de Janeiro, v. 27, n. 65, p. 141-145, jan./mar. 1957.

LEITE, Dante Moreira. Promoção automática e adequação do currículo ao desenvolvimento do aluno. Pesquisa e planejamento, São Paulo, v. 3, n. 3, p. 15-34, 1959.

MAINARDES, Jefferson. A promoção automática em questão: argumentos, implicações e possibilidades. Revista Brasileira de Estudos Pedagógicos, Brasília, v. 79, n. 192, p. 16-29, maio/ago. 1998.

MAINARDES, Jefferson. A organização da escolaridade em ciclos: ainda um desafio para os sistemas de ensino. In: FRANCO, Creso (Org.). Avaliação, ciclos e promoção na educação. Porto Alegre: Artmed, 2001. p. 35-54.

MAINARDES, Jefferson. Organização da escolaridade em ciclos no Brasil: revisão da literatura e perspectivas para a pesquisa. Educação e Pesquisa, São Paulo, v. 32, n. 1, p. 11-30, jan./abr. 2006.

MAINARDES, Jefferson. Reinterpretando os Ciclos de Aprendizagem. São Paulo: Cortez, 2007.

MAINARDES, Jefferson. A organização da escolaridade em ciclos no Brasil: uma análise de pesquisas sobre processos de implementação (1996-2006). Revista Brasileira de Política e Administração da Educação, Porto Alegre, v. 24, n. 1, p. 13-29, jan./abr. 2008.

MAINARDES, Jefferson. A escola em ciclos: fundamentos e debates. São Paulo: Cortez, 2009a. (Questões da nossa época, 137).

MAINARDES, Jefferson. A pesquisa sobre a organização da escolaridade em ciclos no Brasil (2000-2006): mapeamento e problematizações. Revista Brasileira de Educação, Rio de Janeiro, v. 14, n. 40, p. 7-23, jan./ abr. 2009b.

SMITH, Mary Lee; SHEPARD, Lorrie A. What doesn't work: explaining policies in the early grades. Phi Delta Kappan, n. 69, p. 129-134, 1987.

SOUSA, Sandra Zákia; ALAVARSE, Ocimar Munhoz; STEINVASCHER, Andrea; ARCAS, Paulo. Ciclos e progressão escolar: indicações bibliográficas. Ensaio, Rio de Janeiro, v. 11, n. 38, p. 99-114, jan./mar. 2003.

SOUSA, Sandra Zákia; BARRETTO, Elba Siqueira de Sá. Estado do conhecimento: ciclos e progressão escolar (1990-2002) - Relatório Final. São Paulo: USP (MEC/INEP/Comped/PNUD), 2004.

TEIXEIRA, Anísio. Nota preliminar (ao artigo de Moysés Kessel). Revista Brasileira de Estudos Pedagógicos, Brasília, v. 22, n. 56, p. 53-55, 1954. 